Bull. Austral. Math. Soc.

VoL. 50 (1994) [219-223]

\title{
ON STRONG CONVERGENCE OF ARRAYS
}

\section{YONG-Cheng QI}

In this paper we study almost sure convergence for arrays of independent and identically distributed random variables. We obtain a condition under which Marcinkiewicz's strong law holds and get a rate analogous to the law of the iterated logarithm under a condition weaker than Hu and Weber's.

\section{INTRODUCTION}

Assume that $\left\{X_{n}, n \geqslant 1\right\}$ is a sequence of independent and identically distributed (i.i.d.) random variables. Kolmogorov's strong law of large numbers states that

$$
\frac{\sum_{k=1}^{n} X_{k}}{n} \rightarrow a \in \mathbb{R} \text { almost surely as } n \rightarrow \infty \text { if and only if } E\left|X_{1}\right|<\infty,
$$

and Hartman and Wintner's law of the iterated logarithm claims

(2) $\quad \limsup _{n \rightarrow \infty} \frac{\sum_{k=1}^{n} X_{k}}{\sqrt{2 n \log \log n}}=1$ and $\liminf _{n \rightarrow \infty} \frac{\sum_{k=1}^{n} X_{k}}{\sqrt{2 n \log \log n}}=-1$ almost surely

provided that $E X_{1}=0$ and $E X_{1}^{2}=1$.

Now let $\left\{X_{n k}, k=1,2, \cdots, n, n=1,2, \cdots\right\}$ be an array of i.i.d. random variables with $E X_{11}=0$, and for every $n \geqslant 1$ set $S_{n}=\sum_{k=1}^{n} X_{n k}$. Recently, Hu, Moricz and Taylor [2] proved that

$$
\frac{S_{n}}{n} \rightarrow 0 \text { almost surely as } n \rightarrow \infty \text { if and only if } E X_{11}^{2}<\infty,
$$

and $\mathrm{Hu}$ and Weber [3] showed that if $E\left|X_{11}\right|^{4}<\infty$ and $E X_{11}^{2}=1$ then

$$
\limsup _{n \rightarrow \infty} \frac{S_{n}}{\sqrt{2 n \log n}}=1 \text { and } \liminf _{n \rightarrow \infty} \frac{S_{n}}{\sqrt{2 n \log n}}=-1 \text { almost surely . }
$$

(3) and (4) are quite different from (1) and (2), respectively, in the sense that much stronger conditions are needed when the strong law of large numbers and the analogous law of the iterated logarithm are extended from sequences to arrays. In the present paper we extend Marcinkiewicz's strong law to arrays and provide necessary and sufficient condition under which (4) holds.

Received 27th October, 1993

Copyright Clearance Centre, Inc. Serial-fee code: 0004-9729/94 $\$ A 2.00+0.00$. 


\section{MAIN RESUlts}

Throughout the section let $\left\{X_{n k}\right\}$ be an array of i.i.d. random variables and put $S_{n}=\sum_{k=1}^{n} X_{n k}$ for each $n \geqslant 1$.

Theorem 1. Assume $1 / 2<\alpha<\infty$. The following two statements are equivalent:

(a) There exists some $\mu \in \mathbb{R}$ such that

$$
\frac{S_{n}-n \mu}{n^{\alpha}} \rightarrow 0 \text { almost surely as } n \rightarrow \infty ;
$$

(b) $E\left|X_{11}\right|^{2 / \alpha}<\infty$.

Furthermore, if (b) holds, then in case $\alpha>1$, (5) holds for any fixed $\mu \in \mathbb{R}$ and in case $1 / 2<\alpha \leqslant 1$, (5) holds with $\mu=E X_{11}$.

REMARK. When $1 / 2<\alpha \leqslant 1$, the theorem was proved by Hu, Moricz and Taylor [2] under the condition $E X_{11}=0$.

Proof: Note that if $\alpha>1$, then (5) holds for some (or every) $\mu \in \mathbb{R}$ if and only if $S_{n} / n^{\alpha} \rightarrow 0$ almost surely. Thus we can always assume $\mu=0$ when $\alpha>1$. We know that (5) holds if and only if

$$
P\left(\left|S_{n}-n \mu\right|>\varepsilon n^{\alpha}, \text { infinitely often }\right)=0 \text { for all } \varepsilon>0,
$$

which is equivalent to

$$
\sum_{n=1}^{\infty} P\left(\left|S_{n}-n \mu\right|>\varepsilon n^{\alpha}\right)<\infty, \quad \text { for all } \varepsilon>0
$$

by the Borel-Cantelli lemma.

According to Theorem 3 of Baum and Katz [1], for $\alpha>1,(6)$ is equivalent to $E\left|X_{11}\right|^{2 / \alpha}<\infty$ and for $1 / 2<\alpha \leqslant 1,(6)$ is equivalent to $E\left|X_{11}\right|^{2 / \alpha}<\infty$ and $\boldsymbol{\mu}=\boldsymbol{E} \boldsymbol{X}_{11}$. This completes the proof.

THEOREM 2. (4) holds if and only if

$$
E\left|X_{11}\right|^{4}\left(\log ^{+}\left|X_{11}\right|\right)^{-2}<\infty, \quad E X_{11}^{2}=1 \text { and } E X_{11}=0
$$

where $\log ^{+} x=\log (\max (e, x))$.

Proof: Assume first (7) holds. To prove $\limsup _{n \rightarrow \infty} S_{n} / \sqrt{2 n \log n}=1$ almost surely, it suffices to show that for every $\varepsilon>0$,

and

$$
\begin{array}{ll}
P\left(S_{n}>(1+\varepsilon) \sqrt{2 n \log n},\right. & \text { infinitely often })=0 \\
P\left(S_{n}>(1-\varepsilon) \sqrt{2 n \log n},\right. & \text { infinitely often })=1,
\end{array}
$$


that is, by the Borel-Cantelli lemma,

$$
\begin{aligned}
& \sum_{n=1}^{\infty} P\left(S_{n}>(1+\varepsilon) \sqrt{2 n \log n}\right)<\infty \\
& \sum_{n=1}^{\infty} P\left(S_{n}>(1-\varepsilon) \sqrt{2 n \log n}\right)=\infty
\end{aligned}
$$

For $\theta>0$ set $\tilde{S}_{n}=\sum_{k=1}^{n} X_{n k} I\left(\left|X_{n k}\right| \leqslant \theta \sqrt{n \log n}\right)$. It is easily proved from (7) that

$$
\sum_{n=1}^{\infty} P\left(\max _{1 \leqslant k \leqslant n}\left|X_{n k}\right|>\theta \sqrt{n \log n}\right)<\infty
$$

and

$$
n\left|E X_{11} I\left(\left|X_{11}\right| \leqslant \theta \sqrt{n \log n}\right)\right|=n\left|E X_{11} I\left(\left|X_{11}\right|>\theta \sqrt{n \log n}\right)\right| \rightarrow 0 \quad \text { as } n \rightarrow \infty \text {. }
$$

Hence, in order to prove (8), we only need to show that for every $\varepsilon>0$, there exists a $\theta>0$ such that

$$
\sum_{n=1}^{\infty} P\left(\tilde{S}_{n}-E \tilde{S}_{n}>(1+\varepsilon) \sqrt{2 n \log n}\right)<\infty
$$

For any given $\varepsilon>0$, set $\delta=\min (1, \varepsilon), \Delta=((1+\varepsilon) \sqrt{2}) /(1+\delta)$ and take $\theta=\min (\Delta / 5,1)$.

Note that

$$
e^{x} \leqslant 1+x+\frac{1+\delta}{2} x^{2}+\frac{1}{\delta^{4}} x^{4}+\frac{|x|^{5}}{5 !} e^{|x|}, \quad x \in \mathbb{R} .
$$

Putting $\tilde{X}_{11}=X_{11} I\left(\left|X_{11}\right| \leqslant \theta \sqrt{n \log n}\right)-E X_{11} I\left(\left|X_{11}\right| \leqslant \theta \sqrt{n \log n}\right), t=\Delta \sqrt{\log n / n}$ and using (11) we have

$$
\begin{aligned}
& E \exp \left\{t \tilde{X}_{11}\right\} \\
\leqslant & +\frac{1+\delta}{2} t^{2} E X_{11}^{2}+\frac{32}{\delta^{4}} t^{4}(\log n)^{2} E X_{11}^{4}\left(\log ^{+}\left|X_{11}\right|\right)^{-2} \\
& +t^{5}(\log n)^{2} \sqrt{n \log n} \exp (2 \theta t \sqrt{n \log n}) E X_{11}^{4}\left(\log ^{+}\left|X_{11}\right|\right)^{-2} \\
\leqslant & \exp \left\{\frac{1+\delta}{2} t^{2}+\frac{32(\Delta \log n)^{4}}{\delta^{4} n^{2}} E X_{11}^{4}\left(\log ^{+}\left|X_{11}\right|\right)^{-2}+\frac{(\Delta \log n)^{5}}{n^{2(1-\theta \Delta)}} E X_{11}^{4}\left(\log ^{+}\left|X_{11}\right|\right)^{-2}\right\} \\
\leqslant & \exp \left\{\frac{1+\delta}{2} t^{2}+\frac{1}{n^{3 / 2}}\right\}
\end{aligned}
$$


for all large $n$. Therefore, we get that for large $n$

$$
\begin{aligned}
P\left(\tilde{S}_{n}-E \tilde{S}_{n}>(1+\varepsilon) \sqrt{2 n \log n}\right) & \leqslant E \exp \left\{t\left(\tilde{S}_{n}-E \tilde{S}_{n}\right)\right\} \exp \{-(1+\varepsilon) t \sqrt{2 n \log n}\} \\
& \leqslant 2 \exp \{-(1+\varepsilon) \log n\}
\end{aligned}
$$

which guarantees (10). Thus (8) is proved.

To check (9), we use Theorem 1 of Rubin and Sethuraman [5]. Since $E\left|X_{11}\right|^{q}<\infty$ for all $q<4$, we have for $0<\varepsilon<1$

$$
P\left(S_{n}>(1-\varepsilon) \sqrt{2 n \log n}\right) \sim \frac{1}{2(1-\varepsilon) \sqrt{\pi \log n}} n^{-(1-\varepsilon)^{2}}, \text { as } n \rightarrow \infty,
$$

which yields (9).

Similarly, one can show

$$
\liminf _{n \rightarrow \infty} \frac{S_{n}}{\sqrt{2 n \log n}}=-1 \quad \text { almost surely. }
$$

Assume now (4) holds. Obviously, for any $1 / 2<\alpha<\infty, S_{n} / n^{\alpha} \rightarrow 0$ almost surely, as $n \rightarrow \infty$. Hence, by Theorem $1, E X_{11}=0$ and $E\left|X_{11}\right|^{q}<\infty$, for all $q<4$. If we can show that $E X_{11}^{4}\left(\log ^{+}\left|X_{11}\right|\right)^{-2}<\infty$, then we have proved that

$$
\limsup _{n \rightarrow \infty} \frac{S_{n}}{\sqrt{2 n \log n}}=\sqrt{E X_{11}^{2}} \quad \text { almost surely, }
$$

which together with (4) yields $E X_{11}^{2}=1$. Thus, to complete the proof, it suffices to verify that $E X_{11}^{4}\left(\log ^{+}\left|X_{11}\right|\right)^{-2}<\infty$.

It is easy to see that (4) implies

$$
\sum_{n=1}^{\infty} P\left(\left|S_{n}\right| \geqslant 2 \sqrt{n \log n}\right)<\infty
$$

Let $m(X)$ denote a median of the random variable $X$. Then from Rogozin [4],

$$
P\left(\max _{1 \leqslant k \leqslant n}\left|X_{n k}-m\left(X_{11}\right)\right| \geqslant x\right) \leqslant 8 P\left(\left|S_{n}-m\left(S_{n}\right)\right| \geqslant \frac{x}{4}\right), \quad x>0
$$

and additionally, from the central limit theorem, $\frac{m\left(S_{n}\right)}{\sqrt{n \log n}} \rightarrow 0$ as $n \rightarrow \infty$. As a result, we get, for large $n$,

$$
P\left(\max _{1 \leqslant k \leqslant n}\left|X_{n k}\right| \geqslant 16 \sqrt{n \log n}\right) \leqslant 8 P\left(\left|S_{n}\right| \geqslant 2 \sqrt{n \log n}\right),
$$


which coupled with (12) proves

$$
\sum_{n=1}^{\infty} P\left(\max _{1 \leqslant k \leqslant n}\left|X_{n k}\right| \geqslant 16 \sqrt{n \log n}\right)<\infty .
$$

Because $n P\left(\left|X_{11}\right| \geqslant 16 \sqrt{n \log n}\right) \rightarrow 0$ as $n \rightarrow \infty$, we have

$$
\begin{aligned}
P\left(\max _{1 \leqslant k \leqslant n}\left|X_{n k}\right| \geqslant 16 \sqrt{n \log n}\right) & =1-\left(1-P\left(\left|X_{11}\right| \geqslant 16 \sqrt{n \log n}\right)\right)^{n} \\
& =n P\left(\left|X_{11}\right| \geqslant 16 \sqrt{n \log n}\right)(1+o(1)), \text { as } n \rightarrow \infty
\end{aligned}
$$

Consequently, we obtain

$$
\sum_{n=1}^{\infty} n P\left(\left|X_{11}\right| \geqslant 16 \sqrt{n \log n}\right)<\infty
$$

which is equivalent to $E X_{11}^{4}\left(\log ^{+}\left|X_{11}\right|\right)^{-2}<\infty$. Theorem 2 is proved.

\section{REFERENCES}

[1] L.E. Baum and M. Katz, 'Convergence rates in the law of large numbers', Trans. Amer. Math. Soc. 120 (1965), 108-123.

[2] T.C. Hu, F. Moricz and R.L. Taylor, 'Strong laws of large numbers for arrays of rowwise independent random variables', Acta Math. Hungar. 54 (1989), 153-162.

[3] T.C. Hu and N.C. Weber, 'On the rate of convergence in the strong law of large numbers for arrays', Bull. Austral. Math. Soc. 45 (1992), 479-482.

[4] B.A. Rogozin, 'On the existence of exact upper sequences', Theory Probab. Appl. 13 (1968), 667-671.

[5] H. Rubin and J. Sethuraman, 'Probabilities of moderate deviations', Sankhya Ser. A 27 (1965), 325-346.

Institute of Systems Science

Academia Sinica

Beijing 100080

People's Republic of China 